\title{
Revêtements d'hydroxyapatite réalisés par projection plasma : vers de nouvelles fonctionnalités
}

\section{Plasma Sprayed Hydroxyapatite Coatings: Towards new functions}

\author{
Ghislaine Bertrand, David Grossin et Christian Rey \\ Institut Carnot CIRIMAT, UMR CNRS-INPT-UPS 5085, ENSIACET, 4 allée E. Monso, 31030 Toulouse Cedex \\ 4, France
}

\begin{abstract}
Engineered implants for bone and joints replacement very often require coated metallic substrates. The coating is aimed at creating conditions for bone regeneration while still maintaining the bulk mechanical properties of the metallic substrate. Among coating technologies, plasma spray deposition is the most widely used to produce commercial hydroxyapatite coatings. In the present paper, attempts are made to give an overview of latest process developments (suspensions/solutions plasma spray, dc vs rf plasma spray, low energy torch...) as well as materials innovations (doped hydroxyapatite...).
\end{abstract}

\begin{abstract}
Résumé. Dans les stratégies de remplacement/reconstruction des os et des articulations, il est souvent fait appel à des matériaux revêtus. Le matériau de structure permet d'assurer une bonne tenue mécanique et le revêtement doit faciliter et pérenniser l'ancrage dans le site osseux. Dans ce contexte, la technique de projection plasma est largement utilisée pour réaliser industriellement des revêtements d'hydroxyapatite. Nous proposons ici de faire une revue sur les avancées en termes de procédé (projection plasma dc ou rf, de suspensions/solutions, basse énergie,...) couplé à des compositions d'hydroxyapatite modifiées.
\end{abstract}

\section{INTRODUCTION}

Les pathologies (ostéoporose, arthrose, ...) et les traumatismes (fracture du col du fémur,...) du squelette dont les origines peuvent être associées à un vieillissement des populations, mais aussi aux accidents de la route, aux guerres, à certaines activités physiques soutenues, professionnelles ou sportives, sont de plus en plus nombreux (le marché mondial est estimé à 33 milliards de $\$$, en croissance de $7,5 \%$ par an) $[1,2]$.

10 millions d'individus dans le monde portent une prothèse. Remplacer une articulation (hanche, genou, épaule, coude, cheville, doigt, poignet,...) usée ou défaillante ou reconstruire une perte osseuse (sphère crano-maxillo-faciale, ...) nécessite un dispositif médical implantable capable de restaurer les fonctions mécaniques (contraintes mécaniques, usure, fatigue,...) et d'assurer une continuité des fonctions biologiques (croissance des cellules, formation de tissus, ... ), sur le long terme.

La conception des implants et le choix des matériaux s'appuient largement pour les pièces supportant une charge mécanique sur les matériaux métalliques. Ainsi les aciers inoxydables, les alliages à base de cobalt et ceux à base de titane sont proposés commercialement pour les prothèses articulaires, les implants dentaires ou autres pièces de reconstruction. Au-delà de la durabilité et de la fonctionnalité de l'implant, qui sont des objectifs principalement assurés par les propriétés du matériau massif, la réponse biologique dépend, elle, des caractéristiques de la surface du matériau implanté. En effet, l'étape clé concerne la formation de l'interface entre l'os et le matériau implanté qui peut durer plusieurs mois et consiste en la production, par les ostéoblastes, d'une matrice extracellulaire non minéralisée à la surface de l'implant (ostéogénèse de contact) ou sur la surface osseuse (ostéogénèse à distance). Cette matrice, dite ostéoïde, se calcifie par la suite aboutissant à l'ostéo-intégration de l'implant. Dans ce contexte, les métaux et alliages métalliques présentent, en particulier, de mauvaises propriétés d'ostéoconduction/ostéo-induction ainsi qu'une propension à la colonisation bactérienne limitant ainsi leur ancrage et leur intégration. Pour remédier à ces modestes qualités de surface, des modifications morphologiques (texturation 3D) ou physico-chimiques (implantation ionique, greffage, revêtement) ont été proposées. Les céramiques à base de phosphates de calcium $(\mathrm{CaP})$ et en particulier l'hydroxyapatite (HA) $\left(\mathrm{Ca}_{10}\left(\mathrm{PO}_{4}\right)_{6}(\mathrm{OH})_{2}\right)$, dont la composition est relativement proche de celle de l'os sont, à ce titre, utilisées sous la forme de revêtements depuis plusieurs décades, notamment en orthopédie [3]. Ce composé «bioactif» favorise la repousse osseuse au contact et la colonisation par l'os permet d'améliorer la fixation primaire entre l'implant métallique et l'os.

La projection plasma est le procédé le plus répandu au niveau industriel pour la réalisation de ce type de revêtement car elle présente certains avantages dont la facilité de mise en œuvre, de forts rendements de dépôt et une faible température du substrat.

\section{DE LA PROJECTION PLASMA CONVENTIONNELLE À DES MÉTHODES AVANCÉES}

Le procédé de projection plasma consiste à déposer sur un substrat un matériau fondu ou semi-fondu. Le matériau à déposer qui se présente sous forme de poudre est introduit dans le jet d'un plasma (généré par un arc électrique entretenu entre une anode et une cathode) où il est fondu et accéléré vers le substrat. Là il s'étale et se solidifie en quelques ms. Que ce soit la poudre d'hydroxyapatite ou les conditions de projection par plasma atmosphérique 
dc, de nombreuses études ont évalué leur impact sur les caractéristiques du revêtement (adhérence, composition, cristallinité, comportement biologique,...). La maîtrise de la poudre (en termes de distribution granulométrique, densité, composition, pureté) est un facteur permettant de contrôler à la fois la chimie du revêtement mais également ses caractéristiques mécaniques. L'effet des conditions opératoires (conditions plasmagènes, distance de projection, ...) a été étudié grâce à des outils de diagnostic permettant de connaître température, vitesse et position des poudres en vol et confronté à des résultats de simulation numérique $[4,5]$. La température joue un rôle critique dans l'apparition de phases secondaires comme $\mathrm{CaO}$ ou TTCP tandis que la vitesse influence la porosité et les caractéristiques mécaniques du dépôt (microdureté, module d'élasticité). La défaillance du revêtement par éclatement, écaillage, décollement et dissolution a été observée dans des cas cliniques (après plusieurs années d'implantation). Cette défaillance, localisée à l'interface implant/dépôt, a été attribuée à l'existence d'une couche amorphe de phosphate de calcium (ACP) formée lors du refroidissement rapide des particules projetées [6]. Cette zone est fragile et très soluble, ce qui réduit considérablement l'intégrité mécanique de l'interface et en conséquence celle du revêtement lui-même.

Dans le but de limiter ces effets néfastes dus aux hautes températures des développements ont été conduits en minimisant l'énergie de la torche plasma (nouvelle torche basse énergie) et en projetant des apatites plus stables thermiquement comme la chloroapatite [7]. D'autres compositions à base d'hydroxyapatite (Si-HA, $\left.\mathrm{CO}_{3}-\mathrm{HA}, \mathrm{Ag}-\mathrm{HA}, \ldots\right)$ ont également été envisagées dans les dernières années. Notamment Roy et al. ont étudié pour leurs propriétés biologiques, mécaniques et antibactériennes des dépôts d'hydroxyapatite dopée à l'argent, au strontium et au magnésium réalisés par projection plasma inductif à partir de poudres [8]. Bien qu'ayant constaté peu d'effets des dopants sur les caractéristiques microstructurales et mécaniques (taille de grains similaires de l'ordre de 20-25 nm, adhérence identique autour de $17 \mathrm{MPa}$ ), une différence des comportements biologiques a été notée. In vitro l'adhésion et la prolifération des ostéoblastes (hFOB) sur les dépôts Sr-HA sont améliorées. La présence de ce dopant semble aussi améliorer la différentiation cellulaire et son activité (ALP). L'ajout de 2, 4 ou $6 \%$ massique d'argent dans l'hydroxyapatite projetée conduit à des revêtements aux propriétés mécaniques inaltérées (adhérence mesurée à $17 \mathrm{MPa}$ avec une rupture cohésive). La libération de l'argent est faible de l'ordre de $1 \%$ ce qui signifie que l'argent reste présent dans le dépôt et peut agir sur le long terme comme agent antibactérien sans altérer l'activité cellulaire des ostéoblastes jusqu'à $4 \%$. L'addition de strontium à l'apatite dopée en argent permet de contrebalancer l'effet cytotoxique de l'argent vis-àvis des cellules ostéoblastes. Les revêtements préparés avec une buse supersonique (adaptation du système de projection plasma inductif RF standard) sont par ailleurs mieux cristallisés grâce à des temps de séjour dans le plasma plus courts. De plus il est à noter que les conditions plasma retenues (buse supersonique, distance de projection, puissance) ont un effet en particulier sur la taille des cristallites et en conséquence sur l'activité biologique.

Parallèlement aux évolutions de la composition de l'hydroxyapatite visant à conférer au dépôt des caractéristiques biologiques améliorées (intégration et ancrage plus efficient, limitation des infections), le procédé a évolué. L'injection d'une suspension de particules submicrométriques voire nanométriques ou d'un liquide dans le plasma permet de réaliser des poudres ou dépôts finement structurés. Pawlowski et al. ont conduit des travaux sur la réalisation et l'étude de revêtements d'hydroxyapatite par projection plasma de suspensions (synthèse par précipitation puis calcination suivi par un broyage avant la réalisation de la barbotine dans des solvants mixtes eau-éthanol) [9]. Les revêtements ainsi déposés présentent une structure bimodale dont certaines zones sont constituées de HA, TTCP et $\mathrm{CaO}$ tandis que les autres sont constituées uniquement de HA à grains fins (particules initiales consolidées par les passages de la torche plasma). Les revêtements ont été immergés dans une solution simulant le plasma sanguin (solution SBF) et une dissolution préférentielle a été observée. L'adhérence mécanique des revêtements après immersion est diminuée de $20 \%$ environ mais la dureté mesurée par nanoindentation ne change pas. Huang et al. ont utilisé la projection plasma de 《liquides » pour étudier des revêtements à base d'hydroxyapatite [10]. Ils ont alors obtenu, après avoir validé la qualité de leurs revêtements par des essais biologiques, une meilleure performance en termes de prolifération et différentiation d'un dépôt poreux relativement à un dépôt standard. Ils ont ensuite ajouté des dopants ( $\mathrm{Ag}, \mathrm{Si}, \mathrm{Mg}, \mathrm{CO}_{3}^{2-}, \mathrm{Si} / \mathrm{Mg} / \mathrm{CO}_{3}^{2-}$ ) sous forme de sels de nitrate dans leurs suspensions avant projection. Ces travaux ont permis d'apporter la preuve qu'il était ainsi possible de doper une hydroxyapatite avec des éléments en site anionique et/ou cationique en maîtrisant les quantités présentes ainsi que d'obtenir une nanostructuration des dépôts (taille des cristallites de 20 à $50 \mathrm{~nm}$ ). Seul l'effet antibactérien a été testé et lorsque l'argent est associé à une structure poreuse son efficacité a été avérée vis-à-vis de Staphilococcus aureus.

Les derniers développements explorent deux voies : l'une tend à renforcer les caractéristiques mécaniques du dépôt en y incorporant une céramique type zircone et la seconde vise à y associer des agents biologiques (antibiotique lié par un polyCD, protéine morphogénétique BMP, RGD, collagène) [11].

\section{Références}

[1] Rapport «Dispositifs médicaux», DGCIS Prospective, PIPAME, juin 2011, 122p

[2] Fiche marché « le marché des implants orthopédiques», mars 2009, Dep. Finances et Économies du Québec

[3] Paital S.R., Dahotre N.B., Mat. Sc. Eng. R 66 (2009) $1-70$

[4] Cizek J., Khor K.A., Surf. Coat Technol. 206 (2012) 2181 
[5] Dyshlovenko S., Pawlowski L., Roussel P., Murano D., Le Maguer A., Surf. Coat Technol. 200 (2006) 3845

[6] Sun L., Berndt C.C., Gross K.A., Kucuk A., J. Biomed. Mater. Res. (Appl. Biomater.), 2001, 58, 570-592

[7] Demnati I., Parco M., Grossin D., Fagoaga I., Drouet C., Barykin G., Combes C., Braceras I., Goncalves S., Rey C., Surf. Coat. Technol. 206 (2012) 2346-2353
[8] Roy M, Fielding GA, Beyenal H, Bandyopadhyay A, Bose S, Applied Materials \& Interfaces, 2012, 4, 1341-1349

[9] Latka L, Pawlowski L, Chicot D, Pierlot C, Petit F, Surf Coat Technol, 2010, 205, 954-960

[10] Huang Y, Xiao Y, Wang S, Huang Y, Liu X, Wu F, Gu Z, J. of Thermal Spray Tech, 2011, 20(4), 829-836

[11] Taha M., Chai F., Blanchemain N., Goube M., Martel B., Hildebrand H.F., Mat. Sc. Eng. C33 (2013) 2639 